\title{
A Fundamental Lower Bound of Actuating Energy for Broadband Photon Switching
}

\author{
Masahiro Hotta \\ Department of Physics, Faculty of Science, Tohoku University, \\ Sendai, 980-8578, Japan \\ hotta@tuhep.phys.tohoku.ac.jp
}

\begin{abstract}
We derive a universal lower bound of actuating energy $E_{s}$ for broadband photon switching by using an uncertainty relation between time and the negative energy density of quantum fields. We find that broadband photon switching between perfect reflection and perfect transmission over a time $t_{s}$ should satisfy $E_{s} \geq \frac{\hbar}{6 \pi t_{s}}$.
\end{abstract}




\section{Introduction}

Considerable effort has been made to realize a network of long-distance quantum communication by photons. If the range of available wavelengths is very broad, more information can be sent on a quantum communication network using encoding strategies than by monochromatic-beam schemes. For network applications, the technology of photon switching [1] (or similarly, switchable mirrors [2]) are expected to play an important role. From the technological point of view, amount of energy required to operate photon switches should be as small as possible. The energy to actuate the switch depends on the implementation method and, in principle, various kinds of implementation of photon switching may be considered. Then a nontrivial question arises. How can we reduce the actuating energy $E_{s}$ of photon switching in a fundamental level ? In this paper, we consider switching devices, which take an actuating time $t_{s}$, designed to switch between perfect reflection and perfect transmission for incident broadband photons and give a fundamental lower bound of $E_{s}$. During the switching process, the switch body emits undesired photons inevitably due to interactions between the device and the electromagnetic field. The generation of photons by changing boundary conditions is a familiar mechanism in quantum field theory, which cause, for example, the dynamical Casimir effect[3]. We evaluate the minimum work done by the switch, utilizing a gedanken experiment based on a uncertainty relation between time and the negative energy of multi-particle states. Of course, the actuating energy $E_{s}$ is bounded below by the work to generate the undesired photons. Using the evaluation of the work, we show that $E_{s} \geq \frac{\hbar}{6 \pi t_{s}}$. It should be emphasized that the lower bound is satisfied for arbitrary broadband photon switching between perfect penetration and perfect reflection with actuating time $t_{s}$. In this paper, we assume that the photon beams propagate in vacuum and the bandwidth is so broad that arbitrary spatial configuration of electromagnetic field can be realized with a good precision. 


\section{Analysis of modes propagating along the $\mathrm{x}$ axis}

As well known, free electromagnetic field can be expanded as

$$
A^{\mu}=\iiint \sqrt{\frac{\hbar}{(2 \pi)^{3} 2|\mathbf{k}|}} \sum_{\lambda} e_{\lambda}^{\mu}(\mathbf{k})\left[a^{\lambda}(\mathbf{k}) e^{i(\mathbf{k x}-|\mathbf{k}| t)}+a^{\lambda \dagger}(\mathbf{k}) e^{-i(\mathbf{k x}-|\mathbf{k}| t)}\right] d^{3} k,
$$

where we set the light velocity equal to one, $e_{\lambda}^{\mu}(\mathbf{k})$ is polarization vectors and $a^{\lambda}(\mathbf{k}), a^{\lambda \dagger}(\mathbf{k})$ are annihilation and creation operators. In principle, all modes can be coupled to and excited by photon switches during switching operations. The actuating energy $E_{s}$ of a photon switch is bounded below by a sum of inevitable works for all modes to generate undesired excitations. Among the modes, let us concentrate on analysis of the modes which propagate along the $x$ axis because we put the mirror boundary of the switch at $x=0$ in the $y z$ plane. Of course, $E_{s}$ is also bounded below by the work to produce undesired photons of the $x$-axis modes. A beam propagating along the $x$ axis with its crosssection $B$ is described [4] by

$$
A^{\mu}(x, t)=\frac{1}{\sqrt{B}} \sum_{\lambda} e_{\lambda}^{\mu} \phi^{\lambda}(x, t) .
$$

Here $B$ is set large enough. The constant vector $e_{\lambda}^{\mu}$ denotes polarizations of photon and $\lambda$ takes two values of the polarization. The massless fields $\phi^{\lambda}$ satisfy the equation of motion given by

$$
\left[\frac{\partial^{2}}{\partial t^{2}}-\frac{\partial^{2}}{\partial x^{2}}\right] \phi^{\lambda}(t, x)=0
$$

For later convenience, we suppress the superscript $\lambda$ of $\phi^{\lambda}$. It should be noted that $\phi$ has a residual electromagnetic gauge symmetry defined by $\phi \rightarrow$ $\phi+$ const.. The general solution of Eq. (11) is written as a sum of leftand right-moving components: $\phi(x, t)=\phi_{+}\left(x^{+}\right)+\phi_{-}\left(x^{-}\right)$, where $\phi_{+}\left(x^{+}\right)$ denotes the left-moving field and $\phi_{-}\left(x^{-}\right)$the right-moving field with lightcone coordinates $x^{ \pm}=t \pm x$. Note that the usual plane-wave modes give a complete and orthogonal basis. In the left-moving field system, for example, 
an arbitrary function $F\left(x^{+}\right)$of $x^{+}$can be uniquely expanded by use of the modes defined by

$$
u_{\omega}\left(x_{+}\right)=\sqrt{\frac{\hbar}{4 \pi \omega}} e^{-i \omega x^{+}},(\omega \geq 0)
$$

as follows.

$$
F\left(x^{+}\right)=\int_{0}^{\infty} d \omega\left[F_{\omega} u_{\omega}\left(x^{+}\right)+F_{\omega}^{*} u_{\omega}^{*}\left(x^{+}\right)\right] .
$$

The mode functions are orthogonal to each other in terms of the norm defined by

$$
(f, g)=\frac{i}{\hbar} \int_{-\infty}^{\infty}\left[f^{*}\left(x^{+}\right) \partial_{+} g\left(x^{+}\right)-\partial_{+} f^{*}\left(x^{+}\right) g\left(x^{+}\right)\right] d x^{+} .
$$

You can check directly the orthonormality such that

$$
\begin{aligned}
& \left(u_{\omega}, u_{\omega^{\prime}}\right)=\delta\left(\omega-\omega^{\prime}\right), \\
& \left(u_{\omega}^{*}, u_{\omega^{\prime}}\right)=0, \\
& \left(u_{\omega}^{*}, u_{\omega^{\prime}}^{*}\right)=\delta\left(\omega-\omega^{\prime}\right) .
\end{aligned}
$$

Due to this fact, each field is expressed by the plane-wave expansion:

$$
\phi_{d}=\int_{0}^{\infty} d \omega \sqrt{\frac{\hbar}{4 \pi \omega}}\left[a_{\omega}^{d} e^{-i \omega x^{d}}+a_{\omega}^{d \dagger} e^{i \omega x^{d}}\right]
$$

where $d=+$ or - . The creation and annihilation operators $a_{\omega}^{d \dagger}$ and $a_{\omega}^{d}$ obey the standard commutation relations given by $\left[a_{\omega}^{d}, a_{\omega^{\prime}}^{d^{\prime} \dagger}\right]=\delta^{d d^{\prime}} \delta\left(\omega-\omega^{\prime}\right)$ because of Eq.(3)-Eq.(5). By using the annihilation operators, the normalized vacuum state $|0\rangle$ is defined by $a_{\omega}^{d}|0\rangle=0$.

\section{Uncertainty relation between time and neg- ative energy density of quantum field}


The energy-momentum tensor $T_{\mu \nu}$ of the quantum field is defined by adopting the normal operator ordering of $a_{\omega}^{d \dagger}$ and $a_{\omega}^{d}$ in the classical expression of the tensor. It is remarkable that the quantum interference effect between multi-particle states is able to suppress quantum fluctuation of the field and to yield negative energy density of the field [5]. For example, even though the classical energy flux $\left[\partial_{+} \phi_{+}\left(x^{+}\right)\right]^{2}$ of the left-moving field is non-negative, the expectation value of the corresponding quantum flux operator $T_{++}\left(x^{+}\right)=: \partial_{+} \phi_{L}\left(x^{+}\right) \partial_{+} \phi_{L}\left(x^{+}\right)$: can be negative. In spite of the negative energy density, the expectation value of the total energy flux $\int_{-\infty}^{\infty} T_{++}\left(x^{+}\right) d x^{+}$for an arbitrary state remains non-negative because the total flux is given by $\int_{0}^{\infty} \hbar \omega a_{\omega}^{+\dagger} a_{\omega}^{+} d \omega$.

By taking an arbitrary monotonically increasing $C^{1}$ function $f(x)$ of $x \in$ $(-\infty, \infty)$ satisfying $f( \pm \infty)= \pm \infty$, a new set of mode functions

$$
v_{\omega}(x)=\sqrt{\frac{\hbar}{4 \pi \omega}} e^{-i \omega f(x)},(\omega \geq 0)
$$

is obtained that can uniquely expand the field. Actually, we can show that an aribitary function $g(x)$ can be uniquely expanded by the new mode functions. Let us define a function given by

$$
G(x)=g\left(f^{-1}(x)\right),
$$

where $f^{-1}$ is the inverse function of $f$. Then, by the Fourier transformation, $G(x)$ is expressed as

$$
G(x)=\int_{-\infty}^{\infty} d k \tilde{G}(k) \sqrt{\frac{\hbar}{4 \pi|k|}} e^{-i k x},
$$

where $\tilde{G}(k)$ is uniquely determined by $g(x)$ as follows.

$$
\begin{aligned}
\tilde{G}(k) & =\sqrt{\frac{|k|}{\pi \hbar}} \int_{-\infty}^{\infty} d x G(x) e^{i k x} \\
& =\sqrt{\frac{|k|}{\pi \hbar}} \int_{-\infty}^{\infty} d x g\left(f^{-1}(x)\right) e^{i k x}
\end{aligned}
$$

Hence we obtain 


$$
\begin{aligned}
G(x) & =\int_{0}^{\infty} d k \tilde{G}(k) \sqrt{\frac{\hbar}{4 \pi k}} e^{-i k x}+\int_{-\infty}^{0} d k \tilde{G}(k) \sqrt{\frac{\hbar}{4 \pi(-k)}} e^{-i k x} \\
& =\int_{0}^{\infty} d \omega\left[\tilde{G}(\omega) \sqrt{\frac{\hbar}{4 \pi \omega}} e^{-i \omega x}+\tilde{G}(-\omega) \sqrt{\frac{\hbar}{4 \pi \omega}} e^{i \omega x}\right] .
\end{aligned}
$$

Substitution of $f(x)$ into $G$ yields the following result which we really want.

$$
\begin{aligned}
g(x) & =G(f(x)) \\
& =\int_{0}^{\infty} d \omega\left[\tilde{G}(\omega) \sqrt{\frac{\hbar}{4 \pi \omega}} e^{-i \omega f(x)}+\tilde{G}(-\omega) \sqrt{\frac{\hbar}{4 \pi \omega}} e^{i \omega f(x)}\right] \\
& =\int_{0}^{\infty} d \omega\left[\tilde{G}(\omega) v_{\omega}(x)+\tilde{G}(-\omega) v_{\omega}^{*}(x)\right] .
\end{aligned}
$$

The orthonormality in terms of the normal product can be also derived straightforwardly. For example, we can calculate $\left(v_{\omega}, v_{\omega^{\prime}}\right)$ and obtain a right result as follows.

$$
\begin{aligned}
\left(v_{\omega}, v_{\omega^{\prime}}\right) & =\frac{i}{\hbar} \int_{-\infty}^{\infty}\left[v_{\omega}^{*} \partial v_{\omega^{\prime}}-\partial v_{\omega}^{*} v_{\omega^{\prime}}\right] d x \\
& =\frac{1}{2 \pi} \int_{-\infty}^{\infty} e^{i\left(\omega-\omega^{\prime}\right) f(x)} \frac{d f}{d x} d x \\
& =\frac{1}{2 \pi} \int_{-\infty}^{\infty} e^{i\left(\omega-\omega^{\prime}\right) x^{\prime}} d x^{\prime} \\
& =\delta\left(\omega-\omega^{\prime}\right) .
\end{aligned}
$$

In moving from the second line to the third line, we have used the coordinate transformation $x^{\prime}=f(x)$. Similarly, it is proven that

$$
\begin{aligned}
& \left(v_{\omega}^{*}, v_{\omega^{\prime}}\right)=0, \\
& \left(v_{\omega}^{*}, v_{\omega^{\prime}}^{*}\right)=\delta\left(\omega-\omega^{\prime}\right) .
\end{aligned}
$$

For the left-moving field $\phi_{+}$, the new expansion is given by

$$
\phi_{+}\left(x^{+}\right)=\int_{0}^{\infty} d \omega\left[b_{\omega}^{+} v_{\omega}\left(x^{+}\right)+b_{\omega}^{+\dagger} v_{\omega}^{*}\left(x^{+}\right)\right] .
$$


Because of Eq.(7)-Eq.(9), $b_{\omega}^{+\dagger}, b_{\omega}^{+}$are new creation and annihilation operators which satisfy $\left[b_{\omega}^{+}, b_{\omega^{\prime}}^{+\dagger}\right]=\delta\left(\omega-\omega^{\prime}\right)$ and depend linearly on the operators $a_{\omega}^{+\dagger}$ and $a_{\omega}^{+}$. A normalized quantum state $|\Phi\rangle$ defined by $b_{\omega}^{+}|\Phi\rangle=0$ is a squeezed state. For an arbitrary $|\Phi\rangle$, the expectation value of the energy flux of $\phi_{+}$is evaluated as

$$
\left\langle\Phi\left|T_{++}\left(x^{+}\right)\right| \Phi\right\rangle=-\frac{\hbar}{24 \pi}\left[\frac{\dddot{f}\left(x^{+}\right)}{\dot{f}\left(x^{+}\right)}-\frac{3}{2}\left(\frac{\ddot{f}\left(x^{+}\right)}{\dot{f}\left(x^{+}\right)}\right)^{2}\right],
$$

where the dot means a derivative in terms of $x^{+}$. The formula in Eq. (11) can be given in several ways [5]. One simple way is the point splitting method [6] which is based on the relation:

$$
\left\langle\Phi\left|T_{++}\left(x^{+}\right)\right| \Phi\right\rangle=\lim _{\delta \rightarrow 0}\left[\begin{array}{c}
\left\langle\Phi\left|\partial_{+} \phi_{L}\left(x^{+}+\delta\right) \partial_{+} \phi_{L}\left(x^{+}\right)\right| \Phi\right\rangle \\
-\left\langle 0\left|\partial_{+} \phi_{L}\left(x^{+}+\delta\right) \partial_{+} \phi_{L}\left(x^{+}\right)\right| 0\right\rangle
\end{array}\right] .
$$

By substituting the field expansion in Eq.(10) into the first term of the righthand-side of Eq.(12), we obtain the result in Eq.(11).

An important example of negative energy flux is generated by a monotonically increasing $C^{1}$ function $f_{\varepsilon}(x)$ given by

$$
\begin{aligned}
f_{\varepsilon}(x) & =\Theta\left(x_{i}-x\right) x \\
& +\Theta\left(x_{f}-x\right) \Theta\left(x-x_{i}\right)\left[x_{i}-\frac{1}{\sqrt{\varepsilon}}+\frac{1}{\sqrt{\varepsilon}-\varepsilon\left(x-x_{i}\right)}\right] \\
& +\Theta\left(x-x_{f}\right)\left[\frac{\varepsilon}{\left(\sqrt{\varepsilon}-\varepsilon\left(x_{f}-x_{i}\right)\right)^{2}}\left(x-x_{f}\right)+x_{i}-\frac{1}{\sqrt{\varepsilon}}+\frac{1}{\sqrt{\varepsilon}-\varepsilon\left(x_{f}-x_{i}\right)}\right],
\end{aligned}
$$

where $x_{i} \leq x_{f}, \Theta(x)$ is the step function and $\varepsilon=\left(\frac{12 \pi\left|E_{n}\right|}{\hbar}\right)^{2}$ is a nonnegative constant. For the squeezed state $\left|\Phi_{\text {shock }}\right\rangle$ corresponding to $f_{\varepsilon}(x)$, the expectation value of the left-moving energy flux is computed as

$$
\left\langle\Phi_{\text {shock }}\left|T_{++}\left(x^{+}\right)\right| \Phi_{\text {shock }}\right\rangle=-\left|E_{n}\right| \delta\left(x^{+}-x_{i}\right)+\frac{\left|E_{n}\right|}{1-\frac{12 \pi}{\hbar}\left|E_{n}\right| l} \delta\left(x^{+}-x_{f}\right)
$$


where $l=x_{f}-x_{i}(>0)$. The first term of the right-hand side shows the flux of a shock wave with negative energy $-\left|E_{n}\right|$. Because $\int_{-\infty}^{\infty}\left\langle\Phi_{\text {shock }}\left|T_{++}(x)\right| \Phi_{\text {shock }}\right\rangle d x$ is positive, we obtain

$$
\frac{\left|E_{n}\right|^{2} l}{\frac{\hbar}{12 \pi}-\left|E_{n}\right| l} \geq 0 .
$$

Because the numerator is definitely positive, the denominator must be nonnegative, which leads to the inequality

$$
\left|E_{n}\right| \leq \frac{\hbar}{12 \pi l}=\frac{\hbar}{12 \pi\left(x_{f}-x_{i}\right)}
$$

The length $l$ is the arrival interval between two shock waves with negative energy and positive energy. Thus, Eq. (16) shows an uncertainty relation between time and the negative energy of the quantum field.

In the above argument, one might worry about whether nonanalytic behavior of the step function which appears in $f_{\varepsilon}(x)$ leads to a unphysical result or not. However there is no need to worry. In real situations, we can always consider a smooth function $f_{\Lambda, \varepsilon}(x)$ which depends on a physical cutoff parameter $\Lambda$ and approaches $f_{\varepsilon}(x)$ when $\Lambda$ becomes large. Because the bandwidth is assumed so broad that the cutoff $\Lambda$ is big enough, the expression of energy flux in Eq.(14) can be realized in a good precision.

\section{Local fluctuation equivalence between flux- vanishing states}

Next we mention a local fluctuation property of flux-vanishing states. The general form of the function $f(x)$ which satisfies $\left\langle\Phi\left|T_{++}\right| \Phi\right\rangle=0$ is given by

$$
f(x)=\frac{c+d x}{a+b x}
$$

where $a, b, c, d$ are real constants. In a space-time region where the function $f(x)$ takes the form of Eq. (17), there is no difference between the state $|\Phi\rangle$ and the vacuum $|0\rangle$ about quantum field fluctuation, which will be coupled 
to and excited by the switch body in the later analysis. Due to the residual gauge symmetry, the fundamental many-point functions of the input states are not $\left\langle\Phi\left|\phi_{+}\left(x_{1}^{+}\right) \cdots \phi_{+}\left(x_{N}^{+}\right)\right| \Phi\right\rangle$, but are instead $\left\langle\Phi\left|\dot{\phi}_{+}\left(x_{1}^{+}\right) \cdots \dot{\phi}_{+}\left(x_{N}^{+}\right)\right| \Phi\right\rangle$. First of all, it can be easily checked that both the one-point functions of $\dot{\phi}_{+}$ vanish: $\left\langle\Phi\left|\dot{\phi}_{+}\left(x_{1}^{+}\right)\right| \Phi\right\rangle=\left\langle 0\left|\dot{\phi}_{+}\left(x_{1}^{+}\right)\right| 0\right\rangle=0$. The two-point function of $|\Phi\rangle$ is calculated as

$$
\left\langle\Phi\left|\dot{\phi}_{+}\left(x_{1}^{+}\right) \dot{\phi}_{+}\left(x_{2}^{+}\right)\right| \Phi\right\rangle=-\frac{\hbar}{4 \pi} \frac{\dot{f}\left(x_{1}^{+}\right) \dot{f}\left(x_{2}^{+}\right)}{\left(f\left(x_{1}^{+}\right)-f\left(x_{2}^{+}\right)\right)^{2}} .
$$

Substituting eq(17) into eq(18), it is possible to show by explicit calculations that

$$
\left\langle\Phi\left|\dot{\phi}_{+}\left(x_{1}^{+}\right) \dot{\phi}_{+}\left(x_{2}^{+}\right)\right| \Phi\right\rangle=\left\langle 0\left|\dot{\phi}_{+}\left(x_{1}^{+}\right) \dot{\phi}_{+}\left(x_{2}^{+}\right)\right| 0\right\rangle .
$$

Note that the many-point functions of in-field, which describe the initial conditions of the system, can be decomposed via the Wick's theorem into a sum of the products of the two-point functions of the state. Therefore, when the input state is $|\Phi\rangle$, all the local properties in a space-time region where Eq. (17) holds are equal to those of the vacuum input. As an instructive example, let us consider a situation in Figure 1. For the state $|\Phi\rangle$, local quantum fluctuation of a spacetime region $R$ in which the energy flux vanishes $\left(\left\langle\Phi\left|T_{\mu \nu}\right| \Phi\right\rangle=0\right)$ are the same as that of the vacuum state $|0\rangle$. Hence any local event $P$ which takes place in $R$ evolves as if the initial state were the vacuum state $|0\rangle$ (Figure 2), as far as the sequential events originated from event $P$ happen in the region $R$. This property comes from the conformal symmetry of the massless field and is very crucial to derive the lower bound of actuating energy of photon switching in the later discussion.

\section{$5 \quad$ Universal lower bound of actuating energy derived by a gedanken experiment}

We consider now the switching process. A perfect mirror boundary of the switch is located at $x=0$ in the $y z$ plane. The mirror body lies on the left-hand side of the boundary. Until $t=0$, the mirror reflects incident left-moving beams with arbitrary shapes arriving at $x=0$. From $t=0$, 
the mirror and its body gradually become transparent. During the switching interval, the switch generates undesired photons, which energy gives a lower bound of $E_{s}$. Incident beams arriving at $x=0$ after $t=t_{s}$ penetrate completely and continue to propagate freely in the spatial region $x \leq 0$.

By adopting not Schrödinger's but Heisenberg's picture of operators and states, the quantum field can be described by both in- and out- asymptotic fields. In the infinite past, the in-asymptotic field $\phi_{i n}$ evolves freely and in the infinite future the out-asymptotic field $\phi_{\text {out }}$ evolves freely. In the past before the mirror begins to be transparent, plane-wave mode functions of $\phi_{\text {in }}$ are given by

$$
U_{\omega}^{i n}(t, x)=\Theta(x)\left[u_{\omega}\left(x^{+}\right)-u_{\omega}\left(x^{-}\right)\right],
$$

where $u_{\omega}$ is defined by Eq.(2). Note that the mirror boundary condition is satisfied as follows.

$$
U_{\omega}^{i n}(t, 0)=0 .
$$

In the past region, $\phi_{i n}$ is expanded as

$$
\phi_{i n}(t, x)=\int_{0}^{\infty} d \omega\left[A_{\omega}^{i n} U_{\omega}+A_{\omega}^{i n \dagger} U_{\omega}^{*}\right],
$$

where $A_{\omega}^{i n}, \quad A_{\omega}^{i n \dagger}$ are annihilation and creation operators of the in-particles. The in -vacuum state is defined by $A_{\omega}^{i n}|0, i n\rangle=0$. The initial state is $|0, i n\rangle$ for the switching process. In order to solve the scattering problem by the mirror for two shock waves with negative and positive energy density, let us introduce another set of mode functions, which satisfies the mirror boundary condition. For an arbitrary monotonically increasing $C^{1}$ function $f(x)$ of $x \in(-\infty, \infty)$ satisfying $f( \pm \infty)= \pm \infty$, the mode functions are given by

$$
V_{\omega}^{i n}(t, x)=\Theta(x)\left[v_{\omega}\left(x^{+}\right)-v_{\omega}\left(x^{-}\right)\right],
$$

where $v_{\omega}$ is given by Eq.([6) and $V_{\omega}^{i n}(t, 0)=0$. It is possible to expand $\phi_{i n}$ as

$$
\phi_{i n}(t, x)=\int_{0}^{\infty} d \omega\left[B_{\omega}^{i n} V_{\omega}+B_{\omega}^{i n \dagger} V_{\omega}^{*}\right]
$$

A squeezed in-state $|\Phi, i n\rangle$ is defined by $B_{\omega}^{i n}|\Phi, i n\rangle=0$. If we take the same function $f$ as Eq.(13), $|\Phi, i n\rangle$ gives the expectation value of the in-coming energy flux in Eq.(14). If $x_{i}<0$, the negative flux in Eq.(14) is firstly reflected to the right direction by the mirror and propagating freely. As seen 
in Eq.(20), the mirror does not make any tail of the wavepacket and the negative energy flux keeps its shock-wave shape. The switching process of the mirror after $t=0$ does not affect the evolution of the wavepacket with negative energy because the wave runs away at light velocity and causality of the system prevent the switching event from disturbing the wavepacket's evolution. Hence even in the remote future the negative flux propagates to the right with its localized shape and is spatially separated from positiveenergy right-moving excitations. Here a comment is added that if spatial support of the mirror is finite and given by $\left[x_{m}, 0\right]$, then we need another set of in-mode functions given by

$$
U_{\omega}^{\prime i n}(t, x)=\Theta\left(x_{m}-x\right)\left[u_{\omega}\left(x^{-}-x_{m}\right)-u_{\omega}\left(x^{+}+x_{m}\right)\right],
$$

which satisfy the boundary conditions $U_{\omega}^{\prime i n}\left(t, x_{m}\right)=0$. Then $\phi_{i n}$ is expanded as

$$
\begin{aligned}
\phi_{i n}(t, x)= & \int_{0}^{\infty} d \omega\left[A_{\omega}^{i n} U_{\omega}+A_{\omega}^{i n \dagger} U_{\omega}^{*}\right] \\
& +\int_{0}^{\infty} d \omega\left[A_{\omega}^{\prime i n} U_{\omega}^{\prime}+A_{\omega}^{i n \dagger} U_{\omega}^{\prime *}\right] .
\end{aligned}
$$

The in-vacuum state is redefined by $A_{\omega}^{i n}|0, i n\rangle=A_{\omega}^{i n}|0, i n\rangle=0$, and the squeezed in-state $|\Phi, i n\rangle$ is redefined by $B_{\omega}^{i n}|\Phi, i n\rangle=A_{\omega}^{\prime i n}|\Phi, i n\rangle=0$.

In the remote future region after the mirror has been completely removed, two sets of free plane-wave mode functions expands the out-asymptotic field $\phi_{\text {out }}$ as follows.

$$
\begin{aligned}
\phi_{\text {out }}(t, x)= & \phi_{+}^{\text {out }}\left(x^{+}\right)+\phi_{-}^{\text {out }}\left(x^{-}\right) \\
= & \int_{0}^{\infty} d \omega\left[A_{\omega}^{+ \text {out }} u_{\omega}\left(x^{+}\right)+A_{\omega}^{+ \text {out } t} u_{\omega}^{*}\left(x^{+}\right)\right] \\
& +\int_{0}^{\infty} d \omega\left[A_{\omega}^{- \text {out }} u_{\omega}\left(x^{-}\right)+A_{\omega}^{- \text {out } \dagger} u_{\omega}^{*}\left(x^{-}\right)\right],
\end{aligned}
$$

where $A_{\omega}^{ \pm \text {out } \dagger}$ and $A_{\omega}^{ \pm \text {out }}$ are creation and annihilation operators of the outparticles. The out-vacuum state is defined by $A_{\omega}^{ \pm \text {out }} \mid 0$, out $\rangle=0$. It should be stressed that the energy-momentum tensor operators is normal-ordered so as to its expectational value for $\mid 0$, out $\rangle$ vanishes. Hence, the state $|0\rangle$ in Eq.(12) is regarded as $\mid 0$, out $\rangle$. 
Let us consider a gedanken experiment in order to evaluate the inevitable work for the switch to produce undesired photons. Instead of the vacuum state $|i n, 0\rangle$, we take as an input quantum state the squeezed state $\mid \Phi$, in $\rangle$ which satisfies Eq. (14) with $x_{i} \leq 0$ and $x_{f} \geq t_{s}$. The negative flux in Eq. (14) reaches $x=0$ when $t=x_{i}$ and is perfectly reflected. From $t=$ $x_{i}+0$ to $t=0$, no input or output beams reach the switch body. From $t=0$, the mirror boundary and its body gradually become transparent. The switch generates undesired photons, which energy $E_{\Phi}$ gives a lower bound of actuating energy $E_{s, \Phi}$ of the switch in the case. When the positive flux in Eq. (14) reaches $x=0$ at $t=x_{f}\left(\geq t_{s}\right)$, the mirror has been already removed at the point and the flux propagates freely to the left. After $t=t_{s}$, the right-moving flux of the field is described as

$$
\left\langle\Phi, i n\left|T_{--}\left(x^{-}\right)\right| \Phi, i n\right\rangle=-\left|E_{n}\right| \delta\left(x^{-}-x_{i}\right)+\Theta\left(x^{-}\right) \Theta\left(t_{s}-x^{-}\right) T_{s}\left(x^{-}\right),
$$

where $T_{s}\left(x^{-}\right)$denotes the undesired flux induced by the switching. For the total energy of the undesired flux $E_{\Phi}=\int_{0}^{t_{s}} T_{s}\left(x^{-}\right) d x^{-}$, the following inequality holds:

$$
E_{\Phi} \geq \frac{\left|E_{n}\right|}{1-\frac{12 \pi}{\hbar}\left|E_{n}\right|\left|x_{i}\right|},
$$

as will be proven later. Because no input or output energy flux reaches the mirror body from $t=x_{i}+0$ to $t=0$ and Eq. (17) is satisfied around the mirror body during the interval $\left|x_{i}\right|$, the conformal asymmetry argument mentioned above can apply. We are able to regard production and evolution of the undesired photons as event $\mathrm{P}$ and its sequential events of $R$ in Figure 1. Consequently, the actuating energy $E_{s, \Phi}$ of the switch is independent of $|\Phi, i n\rangle$ and exactly equal to the actuating energy $E_{s}$ of the switch with the input vacuum state $|0, i n\rangle$. Therefore $E_{s}$, which we are interested in, is lower-bounded by the undesired photon energy $E_{\Phi}$ of the state $\mid \Phi$, in $\rangle$. It should be noted that the inequality of Eq. (22) is satisfied by all possible input fluxes in Eq. (14). Therefore, the inequality

$$
E_{s} \geq \max _{\left\{\left|E_{n}\right|, x_{f}, x_{i}\right\}} \frac{\left|E_{n}\right|}{1-\frac{12 \pi}{\hbar}\left|E_{n}\right|\left|x_{i}\right|}
$$

must hold. Here $x_{f}, x_{i}$ and $\left|E_{n}\right|$ are constants which appear in Eq. (14). For fixed $x_{f}$ and $\left|E_{n}\right|$, maximizing $\frac{\left|E_{n}\right|}{1-\frac{12 \pi}{\hbar}\left|E_{n}\right|\left|x_{i}\right|}$ is achieved by maximizing $\left|x_{i}\right|$. 
From the uncertainty relation in Eq. (16), $\left|x_{i}\right|$ is bounded above as

$$
\left|x_{i}\right|=\left|x_{f}-x_{i}\right|-\left|x_{f}\right| \leq \frac{\hbar}{12 \pi\left|E_{n}\right|}-\left|x_{f}\right| .
$$

Thus the maximum of $\left|x_{i}\right|$ is $\frac{\hbar}{12 \pi\left|E_{n}\right|}-\left|x_{f}\right|$. Substituting the maximum value into $\frac{\left|E_{n}\right|}{1-\frac{12 \pi}{\hbar}\left|E_{n}\right|\left|x_{i}\right|}$, we obtain

$$
E_{s} \geq \max _{\left\{x_{f},\left|E_{n}\right|\right\}} \frac{\hbar}{12 \pi\left|x_{f}\right|}
$$

Because $\frac{\hbar}{12 \pi\left|x_{f}\right|}$ does not depend on $\left|E_{n}\right|$, the inequality becomes

$$
E_{s} \geq \max _{x_{f}} \frac{\hbar}{12 \pi\left|x_{f}\right|} .
$$

Note that the minimum of $\left|x_{f}\right|$ is $t_{s}$. Hence we obtain a result, ignoring photon polarization:

$$
E_{s} \geq \frac{\hbar}{12 \pi t_{s}} .
$$

In the real situations, photon beams have two massless fields corresponding to two polarizations. Hence, the lower bound of $E_{s}$ should be doubled:

$$
E_{s} \geq \frac{\hbar}{6 \pi t_{s}} .
$$

This result is our main result. The bound holds for the inverse operation to switch perfect transmission to perfect reflection. Because the photon switch can be coupled to other electromagnetic modes propagating to directions different from the $x$ axis, the switch may generate undesired photons in the other modes. We have also neglected the contribution of undesired leftmoving excitations which might be generated during the switching. However, even if these additional contributions exist, the bound of Eq. (28) remains valid because the contributions just increase $E_{s}$. Therefore we can conclude that the bound in Eq.(28) is universal. 


\section{Inevitable work to produce undesired pho- tons}

We now consider the inequality in Eq. (22). It is enough to discuss the out- asymptotic region in the remote future, where the negative flux and additional flux produced by the mirror switch make free evolution. Thus no need to take account of any boundary conditions in the whole space $(-\infty, \infty)$. This inequality comes from a fact that if we are given a negative-energy flux in some spatial region, there must exist positive-energy flux in other regions in order to guarantee the positivity of total energy in the whole space. Let us take an arbitrary out-state $\mid \Psi$, out $\rangle$ of $\phi_{-}\left(x^{-}\right)$which satisfies

$$
\left\langle\Psi, \text { out }\left|T_{--}\left(x^{-}\right)\right| \Psi, \text { out }\right\rangle=-\left|E_{n}\right| \delta\left(x^{-}\right)
$$

for $-\infty<x^{-}<L$. Here, the positive constant $\left|E_{n}\right|$ is fixed independent of $\mid \Psi$, out $\rangle$. The states $\mid \Psi$, out $\rangle$ are not restricted to in-squeezed states. Eq. (29) can be expressed using $x^{-} \in(-\infty, \infty)$ as

$$
\left\langle\Psi, \text { out }\left|T_{--}\left(x^{-}\right)\right| \Psi, \text { out }\right\rangle=-\left|E_{n}\right| \delta\left(x^{-}\right)+\Theta\left(x^{-}-L\right) T_{s}\left(x^{-}-L\right),
$$

where $T_{s}\left(x^{-}-L\right)$ depends on the details of the state $\mid \Psi$, out $\rangle$ and is constrained such that $E=\int_{-\infty}^{\infty}\left\langle\Psi\right.$, out $\left|T_{--}(x)\right| \Psi$, out $\rangle d x$ is positive. We use the Lagrange multiplier method to find a quantum state $\mid \Psi$, out $\rangle$ which minimizes the total energy $E$ with a constraint in Eq. (29). This requires minimizing the quantity $I$, defined by

$$
\begin{aligned}
I & =\int_{-\infty}^{\infty}\left\langle\Psi, \text { out }\left|T_{--}(x)\right| \Psi, \text { out }\right\rangle d x+\int_{-\infty}^{L} \eta(x)\left[\left\langle\Psi, \text { out }\left|T_{--}(x)\right| \Psi, \text { out }\right\rangle+\left|E_{n}\right| \delta(x)\right] d x \\
& +\lambda[\langle\Psi, \text { out }| \Psi, \text { out }\rangle-1],
\end{aligned}
$$

where $\eta(x)$ and $\lambda$ are multipliers. Because we do not need any conditions on the energy flux at $x=L$, we set $\eta(L)=0$. From the variation of $I$ in terms of $\lambda$, we get the normalization condition of the state $\mid \Psi$, out $\rangle$. From the variation of $I$ in terms of $\eta(x)$, Eq. (29) is reproduced. The variation of $I$ in terms of $\mid \Psi$, out $\rangle$ leads to the eigenvalue equation

$$
F \mid \Psi, \text { out }\rangle=-\lambda \mid \Psi, \text { out }\rangle
$$


where $F$ is a Hermitian operator defined by

$$
F=\int_{-\infty}^{\infty}[1+\eta(x) \Theta(L-x)] T_{--}(x) d x
$$

The operator $F$ can be diagonalized by a method given by Flanagan [7]. We define a $C^{1}$ function $f(x)$ such that

$$
f(x)=x
$$

for $x \in(L, \infty)$ and

$$
f(x)=L-\int_{x}^{L} \frac{d u}{1+\eta(u)}
$$

for $x \in(-\infty, L)$. Assuming a monotonic increase of $f(x)$ and $f(-\infty)=-\infty$, which will be justified later, we can expand the right-moving field $\phi_{-}$such that

$$
\phi_{-}\left(x^{-}\right)=\int_{0}^{\infty} d \omega \sqrt{\frac{\hbar}{4 \pi \omega}}\left[b_{\omega}^{R} e^{-i \omega f\left(x^{-}\right)}+b_{\omega}^{R \dagger} e^{i \omega f\left(x^{-}\right)}\right],
$$

where $b_{\omega}^{R \dagger}, b_{\omega}^{R}$ are creation and annihilation operators. The operator $F$ can be rewritten [7] as

$$
F=\int_{0}^{\infty} \hbar \omega b_{\omega}^{R \dagger} b_{\omega}^{R} d \omega-\frac{\hbar}{12 \pi} \int_{-\infty}^{\infty}\left(\partial_{x} \sqrt{1+\eta(x) \Theta(L-x)}\right)^{2} d x
$$

For a fixed $\eta(x)$, the normalized eigenstate $|v a c, \eta\rangle$ with the minimum eigenvalue of $F$ satisfies $b_{\omega}^{R}|v a c, \eta\rangle=0$. This simplifies the problem because the state $|v a c, \eta\rangle$ is a squeezed state in the meaning mentioned above. Its generating function is denoted by $f_{\eta}(x)$. We can find a function $f_{\eta}(x)$ which generates plain-wave mode functions for both $x>L(>0)$ and $x<0$ and satisfies Eq. (29), as follows:

$$
\begin{aligned}
f_{\eta}(x) & =\Theta(x-L) x \\
& +\Theta(L-x) \Theta(x)\left[\frac{1}{\rho^{2}(L-x)+\rho}-\frac{1}{\rho}+L\right] \\
& +\Theta(-x)\left[\frac{x}{(\rho L+1)^{2}}+\frac{1}{\rho^{2} L+\rho}-\frac{1}{\rho}+L\right],
\end{aligned}
$$


where the constant $\rho$ is defined by $\rho=\frac{\left|E_{n}\right|}{\frac{\hbar}{12 \pi}-\left|E_{n}\right| L}$. It is easy to check that this function $f_{\eta}(x)$ is a monotonically increasing $C^{1}$ function with $f_{\eta}( \pm \infty)= \pm \infty$. The corresponding squeezed state $|v a c, \eta\rangle$ takes a minimum of $E$. The energy flux of $|v a c, \eta\rangle$ is calculated as

$$
\left\langle v a c, \eta\left|T_{--}\left(x^{-}\right)\right| v a c, \eta\right\rangle=-\left|E_{n}\right| \delta\left(x^{-}\right)+\frac{\left|E_{n}\right|}{1-\frac{12 \pi}{\hbar}\left|E_{n}\right| L} \delta\left(x^{-}-L\right) .
$$

By integrating the second term in the right-hand-side of Eq. (36), it is shown that the minimum of $E_{\Phi}$ is given by $\frac{\left|E_{n}\right|}{1-\frac{12 \pi}{\hbar}\left|E_{n}\right| L}$. By substituting $L=\left|x_{i}\right|$, Eq. (22) is exactly derived.

\section{Acknowledgments}

I would like to thank M. Ozawa, M. Morikawa and A. Shimizu for useful discussions. This research was partially supported by the SCOPE project of the MIC.

\section{References}

[1] S. E. Harris and Y. Yamamoto, Phys. Rev. Lett. 81, 3611 (1998).

[2] J. S. Huiberts et al., Nature 380, 231 (1996).

[3] For a recent review, V.V.Dodonov, in M.W.Evans (Ed), Modern Nonlinear Optics, Advances in Chemical Physics Series, Willey, New York, Vol 119, Part 1,309(2001).

[4] "Quantum Noise" by C. W. Gardiner(Springer Series in Synergetics) 8.1.7 p237.

[5] N. D. Birrell and P. C. W. Davies, Quantum Fields in Curved Space (Cambridge Univ. Press, 1982).

[6] L.H. Ford, Proc.R.Soc.Lond.A.364,227 (1978).

[7] E. E. Flanagan, Phys. Rev. D56, 4922 (1997). 


\section{Figure Captions}

Figure 1: For the state $|\Phi\rangle$, local quantum fluctuation of a spacetime region $R$ in which the energy flux vanishes $\left(\left\langle\Phi\left|T_{\mu \nu}\right| \Phi\right\rangle=0\right)$ are the same as that of the vacuum state $|0\rangle$. Hence any local event $P$ which takes place in $R$ evolves as if the initial state were the vacuum state $|0\rangle$, as far as the sequential events originated from $P$ happen in the region $R$.

Figure 2: $P$ in the state $|0\rangle$ evolves in the same way of the $|\Phi\rangle$ case, as

far as the sequential events originated from $P$ happen in the same region of $R$ of Figure 1. 


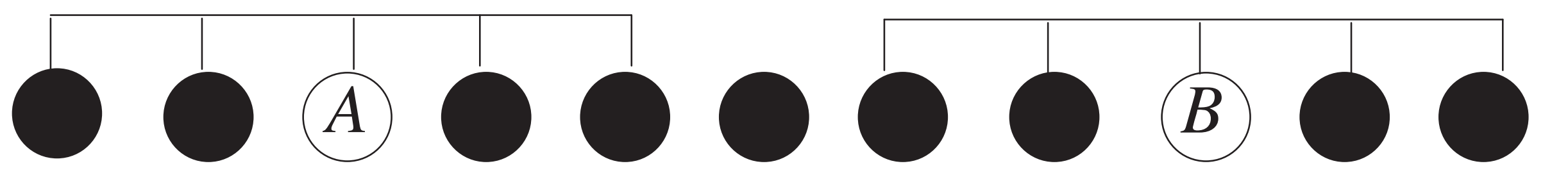

$$
\begin{array}{ll}
\sum_{\mu=0,1}(-1)^{\mu} P_{A}(\mu) & V_{B}(\mu) \\
\hline \text { local measurement } & \begin{array}{l}
\text { local unitary operation } \\
\text { dependent on the measurement result }
\end{array}
\end{array}
$$

average energy input

Classical Channel

$$
\mu=0,1
$$

$$
E_{A} \longrightarrow \text { Quantum Energy Teleportation } E_{B}
$$

Figure 1 